\title{
Chemical Bonds Visualization using Particle Effect and Augmented Reality
}

\author{
Christyowidiasmoro $^{1}$ and Surya Sumpeno ${ }^{2}$
}

\begin{abstract}
Augmented Reality (AR) is a research area that allow to overlay multimedia contents such as text and visual information onto real world through camera views. AR can be used to as an educational assistance to help student acquire a deep conceptual understanding and spatial intuition. The course material can be enriched by viewing multimedia content, as well as in tabletop AR environment. We present this paper an AR system for teaching chemical problem solving especialy chemical bonds to highschool students. Our system is built based on inexpensive webcams and open-source library. Chemical bonds are visualize through marker. By combine different markers, student can get different result and visualization. A survey was conducted after experience the AR chemical bonds, show great acceptance of system and visualization.
\end{abstract}

Keywords_augmented reality, educational assistance, chemistry, chemical bonds, visualization.

\section{INTRODUCTION}

$\mathrm{T}$ he growth of computer technology currently is very fast. Those technologies have many role in various aspect of life. The growth of computer technology can also be used to improve and enhance the teaching and learning process to become more efficient and effective. Chemistry is one of the subjects considered difficult and less desirable by most high school students (Djojonegoro, 1995). One reason is that the chemical has a lot of abstract concepts that are difficult to understand and imagine. If the concepts can be made into real, those can be so easily captured by the student, then the problem can be better understood.

In an effort in that direction, there are already chemistry handbook with a colour images, and exercise in the school laboratory. However, not all problems can be understood by reading chemistry books or experiments in laboratories, such as the atomic structure and chemical bonding. Moreover, the use of confined laboratory at school cannot be done at any time and any place. So it takes a new instructional media in order to improve understanding of concepts appropriately and effectively.

Augmented Reality (AR) as shown in Figure 1 is growing very rapidly thereby enabling the development of applications in various fields, including education. Nowadays, a lot of literature that suggests the possibility of the use of AR in education. AR can be applied in the teaching of geometry, spatial relationships between planets, atomic, molecular structure, and much more. AR is used to help visualize abstract concepts to enhance understanding in describing an object model.

Given the chemical is a relatively abstract concept, then to display animated 3D objects, is expected to be a solution to the lack of high school students' interest towards chemistry subjects. In this paper, will be formulated how to make media interactive chemistry subjects as a learning media, which is through the medium of Augmented Reality technology. There are several considerations in the process of designing and implementing these technologies which consist of

${ }^{1}$ Christyowidiasmoro and Surya Sumpeno is with Departement of Electrical Engineering, Faculty of Industrial Technology, Institut Teknologi Sepuluh Nopember, Surabaya, 60111, Indonesia. E-mail: christyowidiasmoro@ee.its.ac.id; surya@its.ac.id. visualization and simulation of the concept structure of atoms, molecules, and chemical bonds. Designing application as an alternative learning media, that attract high school students to learn and can be used practically in the learning process of chemical bonding.

\section{SYSTEM DESIGN}

ARToolkit library was already able to display virtual objects into the real environment, but the resulting image still has many shortcomings (Sukirman , 2011:31), moreover, to show the corresponding simulation objects as desired chemical theory. Unity 3D is able to solve this problem. Unity 3D can help developer in creating a 3D virtual object and rendering. Developer can add a physical element to the 3D object easily such as rigidbody, texture, collision detection, force, and much more.

In our experiment, the application is created using the augmented reality as our medium and utilizing object tracking functions contained in the ARToolkit library. We built out application on top of Unity3D as our rendering engine. We also use UnityAR as a liaison between the ARToolkit with Unity3D. The design of application systems in this study can be explained in Figure 2.

\section{IMPLEMENTATION}

\section{A. Simulation of Atomic Structures}

Figure 3 shows the results of the state of atomic structure. Users put marker sulfur in front of the camera . The camera captures the pattern box then extract features which is a capital letter ' $\mathrm{S}$ ' in the box area. The pattern features are matched with file pattern that has been previously registered, and it found that those pattern are registered with ID number 13 . Our application then take and calculate the relative position of the marker with the camera. Then put six valences which has been mapped to the ID number 13. Then calculated the total value of valence. Since there is only one marker detected which is sulfur marker, then the total value valence is set to six. Because the total valence is not equal to zero and not equal to a multiple of eight, then the current state is set to state atomic structure. 


\section{B. Simulation of Ion Chemical Bonds}

Ion bonding occurs when element of atoms with a small or low ionization energy release their electron valence (forming a cations) and atoms of other elements that have a larger electron affinity (high), catch or receive those electrons. In our examples, the atomic bonds between $\mathrm{Na}$ and $\mathrm{Cl}$ atoms. $\mathrm{Na}$ atom has one valence electron. $\mathrm{Cl}$ atom has seven valence electrons. When both atom are c onnected, $\mathrm{Na}$ atom will release their electron valence and will be captured by the $\mathrm{Cl}$ atom. This is done so that those two atoms will form a stable matter. After that, the atomic $\mathrm{Na}$ and $\mathrm{Cl}$ has total eight electron valence, and can be called as a stable atom. The process of ionic bonding is described in Figure 4.

Our application will look for markers that have the larger and smaller electron valence. Every atom which has smaller electron valence will be given a force to move those electron to the atom which have larger electron valenc. After a collision is detected, the virtual objects which represent previous electron will be replace with a new virtual object which represent a stable atom.

In this ionic bonds simulation, the experiment will be done to the marker $\mathrm{Na}$ and $\mathrm{Cl}$. The geometric position of atom $\mathrm{Na}$ are recorded and compared to the atom $\mathrm{Cl}$. The data can be seen int table 1 .

From the results shown in Table 1, it can be seen that the Na electrons change position in the $\mathrm{x}, \mathrm{y}$, and $\mathrm{z}$. But the most significant change occurs on the $\mathrm{x}$ axis. When time is 0 seconds, the coordinate are (77.5 -22.5 245.7), when time is 5 seconds, the coordinate are (37.9 -24.7 287.0), when time is 10 seconds, the coordinate are (70.3 -24.0 316.5), when the time is 12 seconds, The coordinate are $(-89.7-23.4317 .5)$. It appears that the electron valence of atom $\mathrm{Na}$ move toward the $\mathrm{Cl}$ atom, until collide and then the electrons will stop.

In the simulation of ionic bonds, there are some physical simulation. This physic simulation is already available in Unity3D with help of NVIDIA PhysX library.

Physical components and their component that affect the simulation are:

1) Rigidbody

Object mass parameter will affect gravity when when ionic bonding occur. The size of object mass with electron vallence is proportional to the force that will be received by electrons of atoms.

\section{2) Collider}

This component serves to detect the touch between one object with another object (collision detector). There is a size parameter, which is the diameter of this component.

3) Constant Force

Occurs when an ionic bond, force is given by the largest atomic valence electrons of an atom valence to the smaller one. The parameter is the magnitude of the force in the direction of the $\mathrm{x}, \mathrm{y}$, and $\mathrm{z}$.

\section{Simulation of Covanlent Chemical Bonds}

$\mathrm{T}$ The simulations was performed for covalent bonding to know the form of the double bond which is carried out on bond of $\mathrm{H} 2, \mathrm{O} 2$, and $\mathrm{N} \mathrm{C} \mathrm{Cl}$.

1) One double bond

Bonds between the $\mathrm{H}$ atoms with $\mathrm{H}$ atoms form $\mathrm{H} 2$ molecules . Electron configuration : $1 \mathrm{H}=\bullet$ ) 1 Those two $\mathrm{H}$ atoms bonded requires 1 additional electrons to obtain a stable electron configuration (according to the electron configuration of He). Therefore , to one of those two atoms, one electron will lend to the other so that there is a shared pair of electrons.

From figure 4, it appears that a virtual object according to H2 covalent bond Lewis structure of a covalent bond $\mathrm{H} 2$.

2) Two double bond

Bonds between the $\mathrm{O}$ atoms with $\mathrm{O}$ to form $\mathrm{O} 2$ molecules. Oxygen atom has the electron configuration : $8 \mathrm{O}=\bullet$ ) 2 ) 6 The $\mathrm{O}$ atom has six valence electrons, so in order to obtain a stable electron configuration of each atom $\mathrm{O}$ requires additional electrons by two. Those two $\mathrm{O}$ atom will lend their two electrons to each other, so those two atom will use two pairs of shared electrons.

From Figure 6, it can be seen that the virtual object $\mathrm{O} 2$ covalent bonds in accordance with the $\mathrm{O} 2$ covalent bond Lewis structure.

3) Mixed double bond

Bonds between the $\mathrm{N}$ and $\mathrm{C}$ atoms to form a molecule $\mathrm{Cl} \mathrm{C}, \mathrm{N}, \mathrm{Cl}$

Electron configuration :

$$
14 \mathrm{C}=\bullet \text { ) } 2 \text { ) } 8 \text { ) } 4
$$$$
7 \mathrm{~N}=\bullet \text { ) } 2 \text { ) } 517 \mathrm{Cl}=\bullet \text { ) } 2 \text { ) } 8 \text { ) } 7
$$

The $\mathrm{O}$ atom has six valence electrons, so in order to obtain a stable electron configuration for each atom $\mathrm{O}$ requires additional electrons by 2 . Those two $\mathrm{O}$ atom will lend 2 electrons to each other, so those two $\mathrm{O}$ atom will use two pairs of shared electrons.

From figure 7, it can be seen that the virtual object that represent the covalent bonds of $\mathrm{C} \mathrm{N} \mathrm{Cl}$ in accordance with the $\mathrm{C}-\mathrm{N}-\mathrm{Cl}$ covalent bond Lewis structure.

\section{Physical Element of Chemical Compounds}

1) Water objects

Some molecules and chemical elements often encountered in everyday life. Such as water and smoke, the main constituent elements of both are $\mathrm{H} 2 \mathrm{O}$ and $\mathrm{CO} 2$. The following, we will exxperiment the realistic element of molecular element. Figure 8 shows the results of experiment of $\mathrm{H} 2 \mathrm{O}$ molecules.

On simulation of $\mathrm{H} 2 \mathrm{O}$ molecule was obtained virtual water object through particle. Some of the components that affect the simulation particles are:

a. Particle Emitter

This component has several parameters that need to set the value of water in order to produce a particle effect in accordance with the real object.

The minimum size of particles is set to 20. The maximum size of the particles is set to 35. Minimum lifetime of each particle is set to 4 seconds. The maximum life span of each particle is set to 5 seconds.

b. Particle Animator

This component serves to give effect to the particles by adding style ( force ) to each particle emitted.

Grow size is represent the growth of particles size during his lifetime. As a water object, this parameter - set to value 0.1 . Rnd Force can be used to make smoke rise to the top, to simulate wind, etc. . Random force is added to each frame of the particle. It can be used to make smoke much more like reality. Force is the force applied to the particles every frame. This parameter value is set $(0,-25,-5)$. While damping is the number of particles 
that will be slowed every frame. When the value is smaller than 1, the particle slows down. This parameter is set to value 1 .

\section{c. Particle Renderer}

The parameters of particle renderer component whih is important to make a shape of the smoke object are:

i. Materials, selected 2D transparent image which have a pattern of misty smoke.

ii. Stretch particle, set to 'billboard'

iii. Max particle size, the value of this parameter is set to 5

iv. World particle collider, In this parameter we set the bounce factor and collide factor.

d. Smoke object

Smoke objects was made from two pieces of particles, which is black smoke particles and fire particles. Both are combined so that a smoke virtual object are shown like in figure 9. When tested in Augmented Reality, the smoke virtual object is almost represent the reality.

From simulation of $\mathrm{CO} 2$ molecules, the smoke virtual object is generated. Some components and parameters affecting particle simulation are:

i. Particle Emitter

Parametes - values of the particle emitter from smoke virtual object are shown in Table 4.

ii. Particle Animator

Parameter - values of the particle animator from smike virtual object are shown in table 5 .

iii. Particle Renderer

This component contains the settings how particles will be rendered later. To form a smoke objects, it is required to selected 2D image transparent materials that make up the pattern of smoke clouding. Material used in this research is Smoke which is the default assets that is contained in Unity3D. Stretch Particle parameters are set 'Billboard' and the maximum size of the particle is set to 0.25 .

\section{E. The relevance of educational material}

Educational materials which are used as reference in this experiment are chemistry course from curriculum 2009 retrieved from electronic textbooks (BSE) 'Memahami Kimia SMA / MA Kelas X' written by Irvan Permana. The publisher is 'Pusat Perbukuan Departement Pendidikan Nasional' from national ministry of culture and education. Table 6 show the comparison of our application related to the reference material.

A total of five points, the material can be offered from our application. While the number chemistry material especialy in Atomic Structure and Chemical bonding in high school curriculum 2009 are ten points. So, our application can covers about $50 \%$ of the material structure of atoms and chemical bonds through simulation.

\section{F. Testing of User Interest}

In the testing phase, our application were tested to the subject, the high school students. Testing method using a sample questionnaire survey methods. Respondents try the demo of our application, then the respondent was asked to complete a questionnaire that has been prepared. The Population sample was 43 participants of high school students. All participants took science majors (IPA) in East Java as shown in table 7.

When asked "how much interest the respondents studied chemistry”, as many as 17 respondents (39\%) responded very interested, 20 respondents (46\%) responded quite interested, and 6 respondents (14\%) responded less interested. And when asked "if they were more interested in studying chemistry after using our application”, a total of 25 respondents (58.1\%) responded more interested, while 18 respondents (41.9 $\%)$ responded not more interested.

From the data obtained the results of the survey, respondents were previously less interested in studying chemistry subjects, after using our application they still remain less interested. However, respondents were previously quite interested and very interested in studying chemistry, after using this software their average interest is increased.

When asked "which is the most interesting scenario in our application", 18 respondents answered the atomic structure, 3 respondents answered covalent bonds, 14 respondents answered the realistic elements of chemical molecules, 4 respondents answered structure of atoms and molecules realistic elements, and 2 respondents answer all interesting scenarios. Figure 10 illustrates the results of the survey.

\section{V.RESULT}

\section{A. Vibration and Pressure of Normal Valve}

From our experiment, we can take a few conclusions :

1. Based on the results of a survey the reality elements of molecule chemical compounds received the highest result which is $48 \%$.

2. Physical components that affect the results of the simulation of Ion bond are rigid body and collider.

3. Particle system components that affect the results of the water test object is Particle Emitter, Particle Animator, Renderer and Particle Collider

4. Particle system components that affect the results of the test object is a smoke Particle Emitter, Particle Animator, and Particle Renderer.

5. Compared to the unmodified one, the modified discharge valve seat increases the discharge pressure. This implies that the opening of a discharge valve happens earlier.

6. Generally speaking, compared to the discharge valve seat as supplied by the OEM, the present of a central torus hole makes the vibration level lower and increases a discharge pressure.

\section{REFERENCES}

[1]. Azuma, Ronald T. - A Survey of Augmented Reality? , In Presence: Teleoperators and Virtual Environments 6, 4 (August 1997), 355-385.

[2]. Permana, Irvan, Understanding Chemical SMA / MA Class X, Center of Books Ministry of National Education, Jakarta: PT. Intan Pariwara, 2009.

[3]. O'Brien, James F. Graphical Modeling and Animation of Fracture, Georgia Institute of Technology, Georgia, USA, 2000.

[4]. Sukirman, Simulation Objects / Physical Objects Using Physic Engine in Augmented Reality Environment, Institute of Technology, Surabaya, Indonesia, 2011.

[5]. Zulkarnaen, Rizky, 3D Model Viewer Application Design Interactive Web-Based Augmented Reality Technology, University of North Sumatra, Medan, Indonesia, 2010. 
[6]. Lopez , Ria Masniari et al, Sampling Techniques in Implementing Research , INFO KSEHATAN, Volume C71, Number 2, March 2003.

[7]. ARToolKit Documentation. $<\mathrm{URL}$ : http://www.hitl.washington.edu/artoolkit/documentation/> September 2011

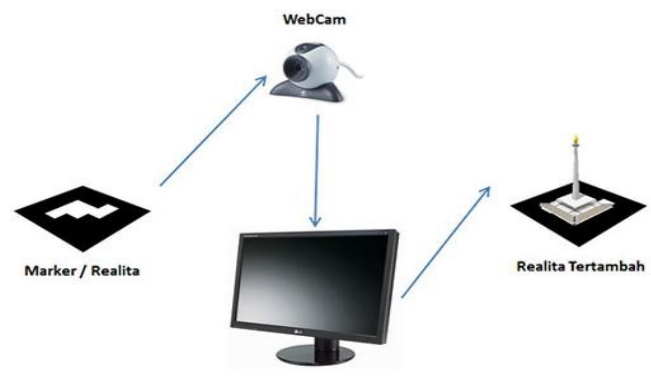

Figure. 1. The Concept of Augmented Reality

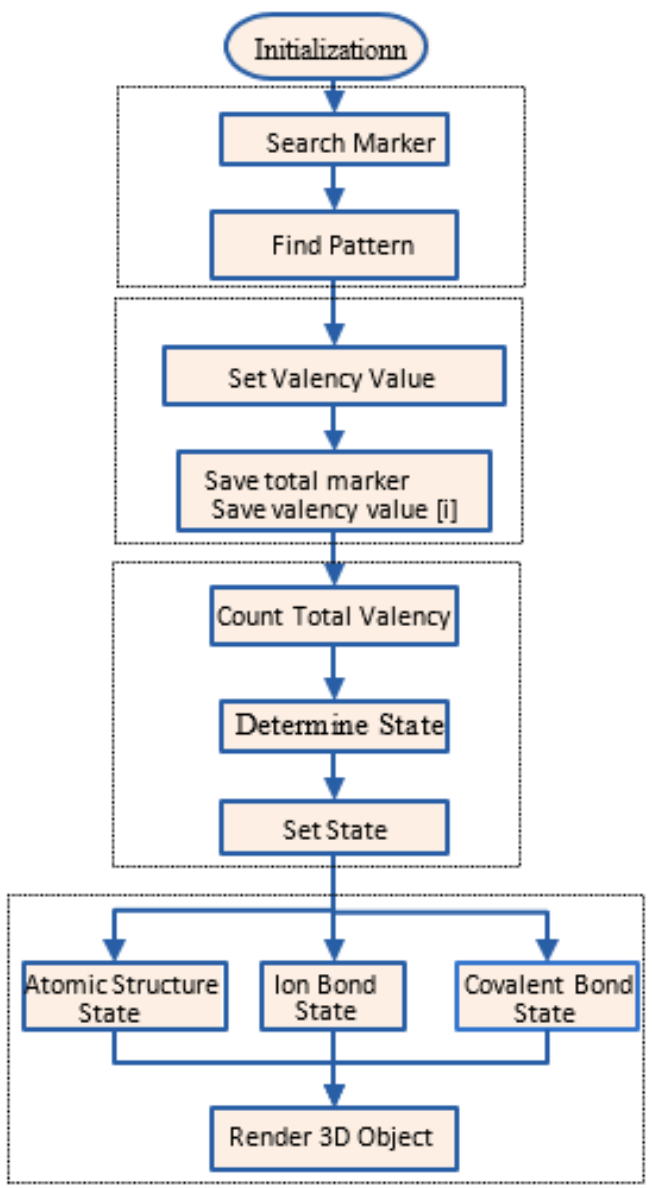

Figure. 2. Flowchart of Our System
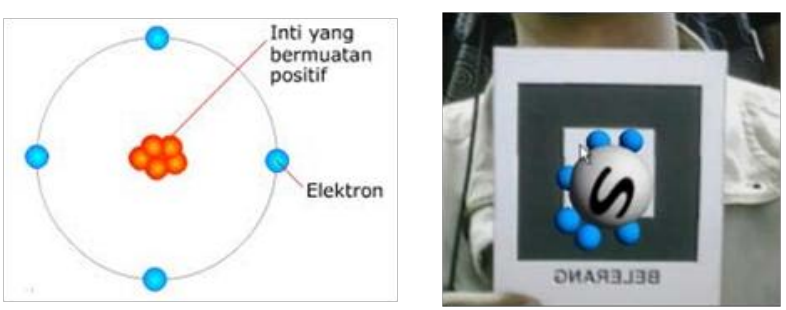

Figure. 3. Atomic Structures

(a) Rutherford atomic model

(b) Structure of Sulfur Atom (S) using augmented reality
[8]. Unity3D Documentation. <URL: Http://unity3d.com/support/ docummentation/ >, September 2011.
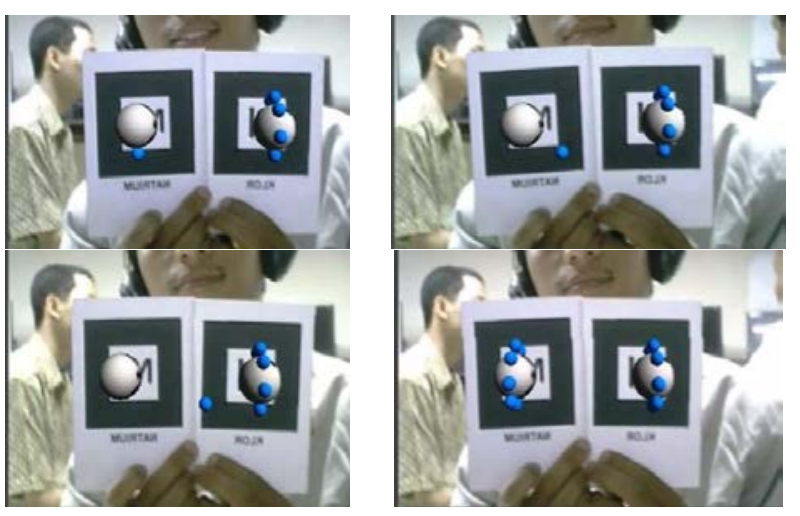

Figure. 4. Simulation $\mathrm{NaCl}$ ionic boding through media Augmented Reality

Lewis structural formula : $\mathrm{H}-\mathrm{H}$

Chemical formula : H2

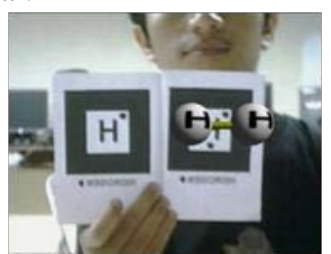

Figure. 5. Covalent bond of H2 using Augmented Reality

Lewis Structural formula : $\mathrm{O}=\mathrm{O}$

Chemical formula : O2

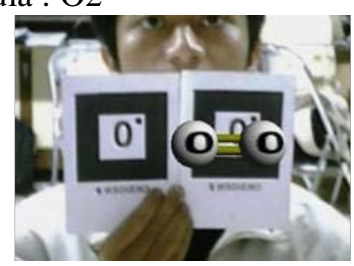

Figure. 6. Covalent bond of O2 using Augmented Reality

Lewis structural formula : $\mathrm{N}=\mathrm{C}-\mathrm{Cl}$

Chemical formula : NCCl

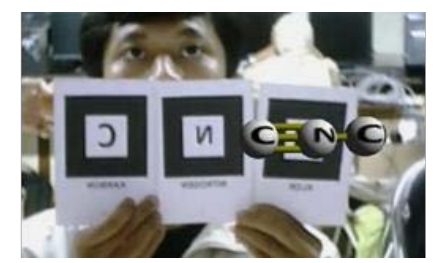

Figure. 7. Covalent bond of $\mathrm{C}-\mathrm{N}-\mathrm{Cl}$ using Augmented Reality

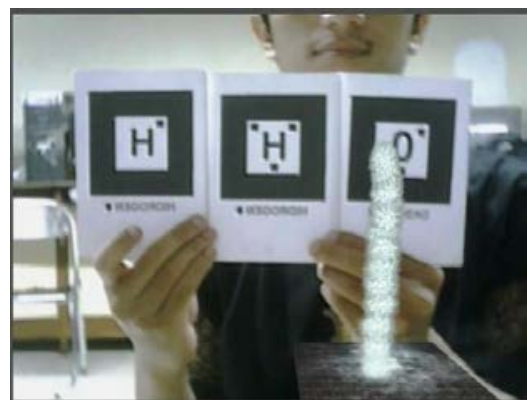

Figure. 8. The simulation of realitic element of covalent bond of $\mathrm{H} 2 \mathrm{O}$ using Augmented Reality 


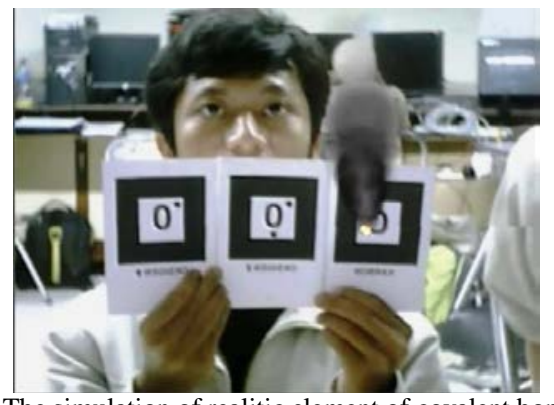

Figure. 9. The simulation of realitic element of covalent bond of $\mathrm{CO} 2$ using Augmented Reality

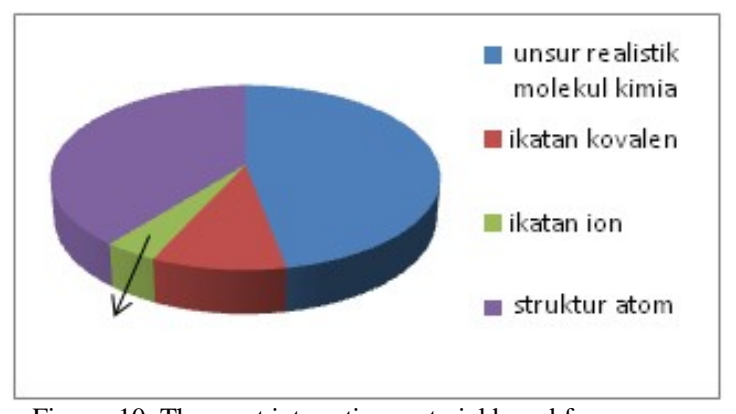

Figure. 10. The most interesting material based from survey

TABLE 1

THE POSITION OF ELECTRONS AND ATOMS WHILE IONIC BONDS SIMULATION

\begin{tabular}{|c|c|c|c|c|c|c|}
\hline \multirow{2}{*}{$\begin{array}{l}\text { Average } \\
\text { time } \\
\text { (second) }\end{array}$} & \multicolumn{3}{|c|}{$\begin{array}{l}\text { Geometric Position of } \\
\mathrm{Na}\end{array}$} & \multicolumn{3}{|c|}{$\begin{array}{l}\text { Geometric Position of } \\
\qquad \mathrm{Cl}\end{array}$} \\
\hline & $x$ & y & $z$ & $x$ & $y$ & $z$ \\
\hline 0 & 77,5 & $-22,5$ & 245,7 & 120,3 & 27,4 & 259,1 \\
\hline 1 & 75,2 & $-22,8$ & 252,2 & 102,8 & $-26,7$ & 274,2 \\
\hline 2 & 69,8 & $-23,4$ & 260,9 & $-115,9$ & $-21,6$ & 263,0 \\
\hline 3 & 60,7 & $-23,9$ & 271,1 & $-110,0$ & $-21,7$ & 262,1 \\
\hline 4 & 47,9 & $-24,4$ & 281,0 & $-111,8$ & $-18,1$ & 264,8 \\
\hline 5 & 37,9 & $-24,7$ & 287,0 & $-110,8$ & $-17,2$ & 265,0 \\
\hline 6 & 10,9 & $-24,9$ & 299,2 & $-115,4$ & $-19,6$ & 262,1 \\
\hline 7 & 2,2 & $-24,9$ & 302,3 & $-110,6$ & $-17,5$ & 269,1 \\
\hline 8 & $-19,6$ & $-24,9$ & 308,5 & $-111,6$ & $-17,9$ & 263,6 \\
\hline 9 & $-49,5$ & $-24,4$ & 314,2 & $-108,2$ & $-16,4$ & 264,6 \\
\hline 10 & $-70,3$ & $-24,0$ & 316,5 & $-107,1$ & $-14,8$ & 265,6 \\
\hline 11 & 89,7 & $-23,4$ & 317,5 & $-107,4$ & $-16,3$ & 265,1 \\
\hline 12 & $-89,7$ & $-23,4$ & 317,5 & $-106,0$ & $-18,1$ & 262,5 \\
\hline
\end{tabular}

TABLE 2 .

PARTICLE EMITTER PARAMETER VALUES TO GENERATE WATER OBJECTS

\begin{tabular}{ll}
\hline \hline Parameter & Value \\
\hline Min Size & 20 \\
Max Size & 35 \\
Min Energy & 4 \\
Max Energy & 5 \\
Ellipsoid & $4,2,1$ \\
\hline \hline
\end{tabular}

TABLE 3 .

PARTICLE ANIMATOR PARAMETER VALUES TO GENERATE WATER OBJECTS

\begin{tabular}{ll}
\multicolumn{2}{c}{ OBJECTS } \\
\hline \hline Parameter & Value \\
\hline Grow Size & 0.1 \\
Rnd Force & $0,0.7,0$ \\
Force & $0,25,-5$ \\
Damping & 1 \\
\hline \hline
\end{tabular}

TABLE 4.

PARTICLE EMITTER PARAMETER VALUES TO PRODUCE SMOKE VIRTUAL OBJECT

\begin{tabular}{ll}
\hline \hline Parameter & Value \\
\hline Min Size & 30 \\
Max Size & 35 \\
Min Energy & 3 \\
Max Energy & 5 \\
Ellipsoid & $1,0,1$ \\
\hline \hline
\end{tabular}

TABLE 5.

PARTICLE ANIMATOR PARAMETER VALUES TO GENERATE THE SMOKE OBJECT

\begin{tabular}{ll}
\hline \hline Parameter & Value \\
\hline Grow Size & 0.5 \\
Rnd Force & $0.1,0.1,0.1$ \\
Force & $0,20,0$ \\
Damping & 1 \\
\hline \hline
\end{tabular}

TABLE 6 .

THE EDUCTIONAL MATERIAL THAT CONSIST IN OUR APPLICATION BASED ON REFERENCE MATERIAL

\begin{tabular}{|c|c|c|c|}
\hline No & Sub Chapter & $\begin{array}{l}\text { Simulation in } \\
\text { Our } \\
\text { Application } \\
\end{array}$ & Status \\
\hline 1 & $\begin{array}{l}\text { Nomor atom \& Nomor } \\
\text { massa }\end{array}$ & - & No \\
\hline 2 & Isotop, Isobar, Isoton & - & No \\
\hline 3 & $\begin{array}{l}\text { Massa atom, massa atom } \\
\text { relatif }\end{array}$ & - & No \\
\hline 4 & Konfigurasi elektron & - & No \\
\hline 5 & $\begin{array}{l}\text { Perkembangan model } \\
\text { atom }\end{array}$ & $\begin{array}{l}\text { Ruterford } \\
\text { atom structure }\end{array}$ & Yes \\
\hline 6 & $\begin{array}{l}\text { Susunan elektro yang } \\
\text { stabil }\end{array}$ & Stable atom & Yes \\
\hline 7 & Ikatan Ion & $\begin{array}{l}\text { Ion bond } \\
\text { simulation }\end{array}$ & Yes \\
\hline 8 & Ikatan kovalen & $\begin{array}{l}\text { Covalent } \\
\text { bond } \\
\text { simulation }\end{array}$ & Yes \\
\hline 9 & Ikatan logam & - & No \\
\hline 10 & $\begin{array}{l}\text { Senyawa fisis, senyawa } \\
\text { ion, senyawa kovalen, } \\
\text { dan logam }\end{array}$ & $\begin{array}{l}\text { Physical } \\
\text { element } \\
\text { compound of } \\
\mathrm{H} 2 \mathrm{O} \text { and } \mathrm{CO} 2\end{array}$ & Yes \\
\hline
\end{tabular}

TABLE 7.

RESPONDENT OF OUR SURVEY

\begin{tabular}{ll}
\hline \hline High School & Total Student \\
\hline SMA 1 Pamekasan & 4 \\
SMA 1 Madium & 3 \\
SMA 2 Tuban & 6 \\
SMA 1 Puri Mojokerto & 3 \\
SMA 2 Mojokerto & 3 \\
SMA 3 Malang & 7 \\
SMA 5 Surabaya & 9 \\
SMA 15 Surabaya & 6 \\
SMA 2 Surabaya & 2 \\
SMA Taman Surabaya & 1 \\
\hline \hline
\end{tabular}

